Masuma H. Mammadova, Zarifa G. Jabrayilova

DOI: 10.25045/jpit.v09.i2.01

Institute of Information Technology of ANAS, Baku, Azerbaijan

depart15@iit.ab.az

\title{
OPPORTUNITIES OF THE INTERNET OF THINGS IN THE MONITORING OF THE PHYSIOLOGICAL STATE AND LOCATION OF PERSONNEL ON OFFSHORE OIL PLATFORM
}

The article explores the opportunities of using the Internet of Things to ensure the safety of personnel on offshore oil platform. To this end, the IoT applications and technologies are analyzed for the monitoring of the physiological state and location of personnel. The article presents the opportunities of using IoT with cloud technologies, Big Data technologies, and artificial intelligence for system development which enables to monitor and, if necessary, to make appropriate decisions based on the systematic monitoring of the state of personnel.

Keywords: offshore oil platform, Internet of Things (IoT), portable smart IoT, Radio Frequency Identification, wireless Sensor Networks.

\section{Introduction}

In accordance with the concept of modern management, the most valuable resource of any facility is a human. This factor is particularly relevant to the facilities of health risks, including the offshore oil platform. Sea Platform (SP) is a complex engineering facility designed for excavation of deposits in the seabed, and in the depth of ocean or other water basin and for the extraction of hydrocarbon raw materials.

Noticeably, the work of oil workers and gas producers is related to health risks. Early Preventive Medical checkup, Evaluation, Inspection and Review System is designed to minimize the risks. However, medical examination does not always guarantee that employees will be able to perform their professional activities during their shift (usually within two weeks in SP). Analysis of emergency situations on oil platforms shows that most of them occur due to unexpected deterioration of health, critical fatigue and loss of consciousness of employees. In this regard, monitoring of employees, their health status (temperature, pressure and other physical controls), and location (space) are currently of great importance.

The issues of production and environmental safety, reduction of accidents, injuries and occupational diseases are among the priorities of the State Oil Company of Azerbaijan Republic (SOCAR). SOCAR pays special attention to security, the development, perfection and implementation of security strategy on SP. There are two main aspects of reducing the number of emergency situations (accidents, injuries, etc.), i.e., reducing the gravity center of the complications on the oil platform. The first aspect involves the development and perfection of technical tools and the support of the working environment, the prevention of their wear and, thus, the prevention of accidents on SP. The second aspect is directly related to the safety of personnel, especially its physiological and psychological condition, behavior, performance on SP and their geographical position, and professional activity. This means that, in addition to registering the equipment, raw materials and environmental parameters, it is important to ensure the proper evaluation of safety of the personnel. Despite the close interrelation between them, both aspects have their own scientific and technical specifications.

The subject matter of this paper is the personnel and its physiological safety on SP. In this regard, it is of interest to observe the dynamics of a number of indicators characterizing the state of labor safety in terms of the human factor and based on SOCAR reports. The reports state that, although the company's work on the occupational safety management system has been successfully implemented and substantial costs have been allocated for strengthening security in this area, the accidents, especially deaths have not been prevented. Table 1 provides information on labor protection costs and accidents at SOCAR for 2010-2016 [1]. 
Information on the total number of employees, labor costs and accidents at SOCAR for 2010-2016 [1]

\begin{tabular}{|c|c|c|c|c|c|c|c|}
\hline & 2010 & 2011 & 2012 & 2013 & 2014 & 2015 & 2016 \\
\hline Number of employees & 79569 & 77140 & 70901 & 61088 & 54714 & 52104 & 50735 \\
\hline $\begin{array}{l}\text { Expenses for improving } \\
\text { labor conditions and } \\
\text { improving health }\end{array}$ & 14407463 & 15301879 & 14480000 & 22340030 & 16481275 & 15956211 & 4366814 \\
\hline $\begin{array}{l}\text { Expenses for providing } \\
\text { employees with special } \\
\text { clothing, footwear and } \\
\text { personal protective } \\
\text { equipment }\end{array}$ & 9480500 & 14541559 & 14020000 & 8000657 & 4625091 & 3964089 & 6400676 \\
\hline $\begin{array}{l}\text { Accidents at subordinate } \\
\text { enterprises of the company: }\end{array}$ & 19 & 19 & 13 & 12 & 13 & 9 & 11 \\
\hline including fatal accidents: & 4 & 12 & 4 & 3 & 7 & 3 & 5 \\
\hline Number of deaths: & & & & & 12 & 15 & \\
\hline Number of missing: & & & & & & 19 & \\
\hline Number of injured: & & & & & 18 & & \\
\hline
\end{tabular}

As the table shows, although there is a slight decline in the number of incidents in recent years, the increase in the number of fatalities, missing and injured employees is evident. Unfortunately, one of the events resulted in such a massive death occurred on December 15, 2015 at the "Gunashli" marine platform fire.

According to SP design standards, SP is projected to ensure the module to be at the maximum distance from drilling and is protected with the helicopter platform on the top, whereas the platform is constructed so that the residing module does not suffer from wind [2]. Such design focuses on ensuring the safety of the residing module when there is an accident on SP.

The key point here is related to the human factor, the main reason for the tragedy is the personnel's insufficient instruction on emergency situations on the platform. One of the chief ways to prevent the "dangerous behavior" of a personnel, or more precisely, the human factor on SP, is a systematic monitoring of the physiological and psychological state of an employee and his/her geographical position at the place. The Internet of Things, modern mobile technologies, the Internet of Medical things and numerous ICT technologies open up wide opportunities to strengthen security measures through the real-time monitoring of the health of personnel on SP and systematic monitoring of the location of each employee.

The exploration of the possibilities of the Internet of Things (IoT) to ensure the safety of staff on SP requires first of all the study of the concept and the essence of the "Internet of Things".

\section{The concept of "The Internet of Things"}

The concept of the "Internet of Things" was first used by the English innovator in the field of technology Kevin Ashton in 1999 for the description of the system in which the objects of the physical world were connected to the Internet through transmitters. In the same year, the Massachusetts Institute of Technology established the Radio Frequency Identification (RFID) and Auto-ID Center, and respectively, the concept of IoT has gained a wide range.

The simple idea of the Internet is that surrounding objects or things (such as tablets, smartphones, fitness equipment, home appliances, clothes, cars, manufacturing equipment, medical equipment, medicines, etc.) can be equipped with miniature identifiers and sensors (sensitive devices) and can be connected to the Internet and to each other via wired and wireless (satellite, mobile, Wi Fi and Bluetooth) connections [4, 5]. Availability of the necessary channels allows not only to identify and monitor the parameters of these objects by space and time, but also to manage them. Thus, the "Internet of Things" can be viewed as a global network infrastructure consisting of a large number of devices, interconnected through sensors, communication, 
networking and information technology [6].

From the information-communication point of view, the Internet of things can be typically written as a symbolic formula as follows [7]:

IoT $=$ Sensors $($ Transmitters $)+$ Data + Network + Services

\section{Definition of "The Internet of Things"}

At present, the term "Internet of Things" does not have a single universally accepted definition in literature. Many definitions of IoT focus of its various aspects and features [7-9]. Some of them are listed below.

1. The Gartner analytical company interprets the "Internet of Things" as a network of physical objects based on technology that allows measuring the parameters representing the position of the physical objects as well as their environment, and using and transferring this data. The main aspect of this interpretation is that, despite the notion of "the Internet of Things", the things are often linked via the M2M (machine-to-machine) protocols rather than the Internet. In this case, it is focused on decisions based on the interactions of transmitters, sensors and other "iron" things without the participation of people [10].

2. The Internet of Things - Physical devices connected to the Internet - a global network of "things" equipped with data transmission facilities that enable sensors, transmitters and data collection and share ("communicate" with each other). These facilities are connected with data management, control and processing center access tools [11].

3. The International Telecommunication Union defines the Internet for Thing as a global infrastructure for the information society, providing the opportunity to deliver more complex services by connecting physical and virtual objects based on existing and emerging ICTs [12].

Due to the rapid development of technology, various interpretations of IoT are given, however they are all based on the same concept. According to this concept, IoT is referred to the group of non-computerized devices, equipment, transmitters, and routine tools with computing ability and network access. Most of these devices usually referred to as "intelligent devices" can process and utilize, analyze and share data with the minimal interference of human.

These things interacting and communicating with each other, and sharing information about the environment and responding to environmental processes without human intervention are predicted to be the most active participants of business, information and social processes in the future $[13,14]$.

\section{Portable intelligent Internet of things}

At present, the continuously expanding market of health Internet o things have been applied in many healthcare sectors. Thus, many health centers around the world have been using smart clothes, gadgets for sensing data on key health parameters, climate control platforms in hospitals, applications for transferring health data to physician, and so forth. For interconnection of different devices with different applications, they are combined with different types of networks over the Internet.

Note that various types of health Internet of things are referred to the large number of intellectual devices that are easily accessible in our daily lives. They are portable (in bodies) intelligent Internet things.

Today, there are numerous body-worn wearable devices that measure human physiological parameters [15]. Portable biomedical transmitters are the subset of devices measuring human biological parameters. Portable IoT technologies are typically rigid or flexible, based on ordinary electronics, and are designed for low power consumption. They are able to control the patient's parameters in a natural environment and an arbitrary environment, and to transmit signals and information.

In the market of the health Internet of things presents various portable technologies installed 
on the transmitters that are categorized as "smart clothes" (head covers, space suits, helmets, jackets, trousers, coats, socks, etc.) and "smart things" (glasses, watches, rings, bracelets, sticks, bandages, lens etc.) [16]. "Smart Clothes" can be used to sense and analyze the data related to the physical activity of a person, to control vital health indicators. They can control the state of people working in hazardous conditions, and monitor the location of the patients and their place of residence in emergency situations, and to observe fatigue levels of an oil man, pilot, driver, etc.

"Intelligent things" (gadgets or devices) may include body-worn transmitters, the objects to monitor vital health indicators, to track the location of the staff on SP, and the applications or objects (e.g. smart box for ampule/pills) reminding the stuff, as well as the healthcare personnel observing their physical state (both physicians), smart devices for real-time monitoring of critical health status, transmitters for tracking and transmitting geolocation data.

Note that each wearable smart gadget has certain functional capabilities and is intended to address specific issues. This allows classifying the portable IoTs for their functional capabilities and relevant applications.

Essentially, portable medical IoTs can be grouped as follows:

1) IoTs for monitoring, diagnostics, treatment, care and rehabilitation of personnel;

2) IoTs for supporting healthy lifestyle of staff, including their daily physical activity and physical condition;

3) Transmitters for tracking staff displacement.

First-group devices include measuring and analyzing one or more vital indicators and parameters (cardiac frequency, ECG, arterial pressure (smart tonometer), sugar rate (smart glucometers), respiratory rate) characterizing the functioning of the cardiovascular system, and the transmitters for drug control.

Second-class portable devices are designed for continuous monitoring of the level of human agility (SP employee), and shows the data, pulse rate, distance traveled, calories consumed, and so forth sensed from accelerometers. The sensed data can be transmitted to the employee's smartphone or computer as well as to the medical personnel (physicians) who treat them. The applications specifically designed for the calculation of various human health indicators provide advice to users when they detect abnormalities in these indicators, and notify the healthcare personnel in critical situations (insult, heart attack, epilepsy, etc.) and for emergency medical intervention.

Third-class mobile devices can be used successfully for tracking the location of people at high risk areas, including people from vulnerable categories (elderly, children, people with psychological illnesses).

\section{Intelligent IoT applications}

Success of IoT depends on the applications that improve the daily life of a person. The requirement for the use of certain applications is the presence of transmitters for the transmission of the relevant data set. IoT applications are software applications or software systems designed to handle specific issues [17].

IoT applications play an important role in e-health and are directly used by users and patients. In addition, they enable tracking and monitoring the health of people working in other areas, including those of specific risks. IoT applications supporting one or more health indicators at the same time are shown below.

Glucose level sensing. Blood sugar monitoring enables detecting blood glucose level and nutrition plan, activity, and the time to take medication [18].

At present, IoT-based health monitoring systems are being successfully implemented. However, modern IoT-based continuous monitoring systems for sugar levels are not numerous and existing systems also have some restrictions. In [19], using IoT, the architecture for glucose level monitoring system is developed. The system provides actual information about the amount of glucose in blood, including body temperature and contextual data (e.g. environmental temperature) in real-time mode in 
a timely or affordable format to the users (patients and physicians).

The authors of the article [20] offer the configuration for Internet of m-health (m-IoT) for non-invasive real-time monitoring of glucose providing IPv6-connectivity with medical service providers (physicians) based on the transmitters mounted on the patient's gadgets.

The article [21] introduces a model for sugar monitoring system based on IoT networks comprising the devices for blood collection and glucose level measurement modules, including mobile phones or computers for data transfer.

Electrocardiogram monitoring (ECG). ECG is one of the methods of simple, palliative and informative diagnostics of heart disease. The method is based on the recording of electrical impulses that occur in the heart and its graphic writing in the form of extensions on the special paper film. ECG reflects numerous variations of the certain parts of heart in the form of extensions [22].

In recent years, various studies have focused on the use of IoT in ECG monitoring. IoT technologies are now thought to be quite promising for the acquisition of maximum amount of information related to the activities of cardiovascular system, including ECG monitoring [23, 24]. The article [25] offers IoT-based system for real-time authentication of heart function. The system records the electrical activity of the heart and sends this data to the data analysis center, which detects ECG errors and evaluates the the patient's condition. Another article [26] develops a complex of algorithm for practical detection of ECG signals in IoT environment for continuous monitoring of ECG. In [27], IoT-based applications are developed to write and monitor of ECG, to measure heart rate and provide the graphical description of the heart rhythm, and then to send these data to the databases and web servers. ECG collecting devices cover the transmission of data on the frequency of heart attacks and Arduino microcontrollers. The software is written in Matlab and $\mathrm{C}^{++}$programming languages in order to process and analyze ECG and download it into the database and Web servers.

A new generation Nuubo, offering a wireless and remote platform for heart monitoring, is of great interest. This Spanish company produces, manufactures and sells a portable medical technology portfolio for the diagnosis and rehabilitation of cardiovascular diseases. These tools are based on the ECG wireless remote monitoring platform and incorporate patented biomedical electronic tissue technology (electronic smart tissues), which uses digital technology called BlendFix Sensor [28].

The advantage of Nuubo series transmitters is that they can be installed on a daily basis. Smart Nuubo shirt is equipped with special devices that control the vital condition of the patient and his/her movements. The shirt records ECG, transmits the sensed data through wireless network to the server for analysis, where special software can define the abnormal parameters. The transmitters installed in the shirt regularly collect parameters such as heart rate, arterial pressure, and body temperature. Shirt-mounted transmitters use a GPS network for connectivity and ensure recording of people on the move (moving throughout the SP) [28].

Heart rate. The frequency of heart rates is characterized by the number of rates in the same time and measured in bits per minute. The frequency of heart rates is substantially dependent on context, i.e., it increases after physical exercises and can change due to stress, insomnia, illness and drug intake. The frequency of heart rates is also affected by age and genetics. IoT-based ECG monitoring systems incorporate the measurement of heart rate and pulse rate, as well as the diagnostics of multichannel arrhythmias, myocardial infarction, and so forth. In healthy people, the frequency of heart rates and pulse are equal $[25,26]$.

Blood pressure monitoring. Blood pressure is referred to the Withings in the arterial vessels. The blood pressure in arteries, veins and capillaries differs and is one of the main indicators of the functional status of an organism.

High blood pressure is a risk factor for the development of insidious, cardiovascular, and chronic renal failure, thus, it is important to systematically monitor and check the effects of treatment. At present, there are a large number of different devices, including mobile devices, to 
measure blood pressure.

The relevance of the problem is that blood pressure-measuring devices and a set of mobile phones supported by NFC (Near Field Communication) have to be related so that they will make up a part of blood pressure monitoring on IoT networks. Performance of the blood pressure measuring devices depends on the model connecting them with a mobile computing device (for example, Apple), which realizes wireless data transmission [29]. The article [30] proposes a device equipped with the apparatus for blood pressure measuring with communication modules for collecting and transmitting blood pressure data in IoT networks. The article [31] offers IoT-based Intelligent Terminal, which identifies the location of the portable apparatus for blood pressure monitoring.

Body temperature monitoring. Body temperature monitoring is an integral part of health care. This indicator is a vital parameter for homeostasis support [32].

Traditional method of temperature measurement through mercury thermometers has been recently replaced by more reliable and inexpensive wire and wireless sensor transmitters for the determination of health status. To determine body temperature, the article [20] provides an example of the realization of m-IoT concept, which uses transmitters installed on TelosB Mote platform. Another article presents IoT-based architecture for temperature control system [33]. RFID module and body temperature control module are the key components of the system responsible for the temperature recording and its transmission in the system.

Smartphones for the development of IoT Solutions in healthcare. Recently, smartphone-driven transmitters have been developed. This enhances the essence of smartphones in the development of IoT technologies. Various hardware and software products that transform smartphones into a universal health device have been developed [34]. Modern smartphones support a large number of health applications (diagnostic, reference, commentary, analytical, etc.) [35]. Health applications also use numerous wireless transmitters running on algorithms to analyze images [36].

Smartphones include effective diagnostics of asthma, cystic fibrosis, allergic rhinitis, as well as monitoring of vital indicators as heart rate, blood pressure, respiratory rate, and so on [35]. Health applications for smartphones offer solutions that are not too expensive for both the patient and the wide user audience. Today, there is a tendency of using multifunctional applications to interact with several sensor devices as of ZephyrLIFT series, while the first health applications were designed to interact with one transmitter.

Applications for geographical location tracking. At present, there is a growing need for solutions that enable each person to track the trajectory of traffic and notify the emergency response service immediately about hazardous situations. Modern mobile devices are equipped with information on their geographical location. For example, Corvus-Tracker for Android operating system is designed to track users' mobile devices. This application sends information about users' geographic location to the monitoring system server. The system is also capable to send SOS signals to the specified phone numbers. The complementary function of the system is to create a geographic area, run within a given time, combine several users in one group, and visualize the system data for the user [37]. Applications for "smart" items' tracking are also widespread. Thus, applications supporting Global Positioning System (GPS) provides operational information to the relevant medical personnel about the location of the users (including those with special risks, as objects, children, elderly people, mentally handicapped people, etc.) through pre-installed sim-cards.

Nowadays, modern systems providing the identification of real-time location system (RTLS) are available. Actually, GPS is considered to be the most important RTLS. This satellite-connected navigation system is able to find objects anywhere on the Earth in different weather conditions. The basic principle of the system is to determine the geographical location by measuring the time of receipt of synchronized signals from antenna satellite navigation in any point of the Earth and of the space. This feature of the GPS can be used for the identification of the location of medical part of the ambulance, patients, physicians, as well as the staff on SP, and for the acquisition of the information on the location [38]. 


\section{Technologies used in Health IoTs}

The IoT concept for healthcare is realized through a range of technologies. A brief description of these technologies is shown below.

The instruments that constitute the basis of IoT and allow the integration of physical devices into the digital world include RFID technologies and Wireless Sensor Networks (WSN).

RFID technologies are based on the use of microcircuits that collect information from the devices mounted on the machine or chips installed on the devices. RFID technology enables the transmission of identifiable information wirelessly to the meters via microchips. RFID meters allow identification, monitoring and control of any object automatically integrated with RFIDs [39].

RFID technologies can be used in IoTs to track the movement of staff on SP. By integrating IoT technologies with e-Health solutions, it is possible to assign RFIDs to each staff member on SP and to send data to the center. This allows the staff to access electronic health records, and the sent data is stored on the Health Center database. The physician gets access to health records of a specific person by scanning the RFID tag [40, 41]. RFID tag can register any person on SP or the specific business areas of the platform. This is especially important in extreme situations to get accurate information about people on SP and their location.

Wireless Sensor Networks (WSN) or Ubiquitous Sensor Networks (USN) constitute a technological basis for the realization of the concept of the Internet of things [42]. Wireless Personal Area Network (WPAN) is a distributed network of unserved miniature electronic devices (sensor nodes) that provides collection of data about external environment parameters and transfer them to the processing center based on retranslating them from node to node. All transmitters are linked with interconnected radio-channels located in the air, water and water surface, and inside the body.

Wireless body area network (WBAN) is a wireless computer network which is wearable and portable on body. These devices can be mounted and implanted into the body, mounted to the body in certain conditions, or installed in clothing (e.g. in a pocket) and carried items (e.g., in a bag) that people wear in different places. WBAN system can use wireless network as a gateway to reach greater distances. The wearable devices can be interconnected through the Internet via the gateways. Thus, health workers can access the information online regardless of the patient's locattion [43].

\section{IoT-based medical data transfer technologies}

At present, networking combinations with different forms and able to operate from different distances, and requiring different powers open wide possibilities for IoTs. They may include wireless personal area networks (WPAN), WiFi networks, wireless mesh networks, cellular networks, extremely broad-band networks, and satellite-connected networks.

IoTs are grouped into near and remote activity segments. The near business segment mainly covers the devices linked with connection channels through the use of unlicensed radio communication technologies (Wi-Fi, ZigBee, Bluetooth) covering 100 meters or fixed as a local area network (LAN), PLC (Power Line Communication). The remote activity segment covers the devices connected through cellular networks, unlicensed low-band radio communications technologies (such as LoRa, Sigfox) or satellite technologies.

IoTs are assembled into two groups for distant and remote data transfer. Today there are various portable devices that support medical sensors to collect data. Most of these devices provide connection at a near distance. This can be a link between nodes or sensor nodes, or a gateway aggregating the data from sensors.

If the data is required to be transmitted to the nearest distance, the device can use a Personal Area Network (PAN), as well as wired USB interface presented with wireless data transmission technologies such as BLE (Bluetooth Low Energy), ZigBee, and 6LoWPAN.

$L A N$ can be used when the data is partially transmitted at a far distance (e.g., within a clinic 
or hospital). Wired local networks are often built on Ethernet and optical fiber technologies, while the wireless ones are built on Wi-Fi technology. WiMax, LTE, etc. are used for the organization of a global network (Wide Area Network, WAN) [44]. Over the past two years, the technology has been developed to connect low-powered devices to LPWAN [45].

Data transmission speed and energy consumption are the key factors for the choice of cellular technology in specific cases. BLE, ZigBee, Z-Wave are used in limited-powered devices and comprises the use of gateways for data aggregation and sending them in IP-network [5].

IoT uses the BLE and ZigBee technologies for data transfer at a close distance.

Bluetooth LE technologies. Bluetooth is a wireless technology that provides the data transmission between the devices that are not too far away from each other. This technology allows the communication between the devices within the coverage of 10 meters. One of the significant advantages of Bluetooth $L E$ is its low power consumption and extreme low power consumption in sleep mode. In other words, the device "sleeps" at $99 \%$ of the time and "awakes" for a short period of time, shares data and "re-sleeps" again. In general, $B L E$ is very advantageous in medical applications. It is safe and has low bandwidth, low latency, low power consumption and resistant to hindrances. This standard is recommended for the design of portable healthcare systems.

Bluetooth 5.0 is the newest generation of Bluetooth, allowing for data sharing between devices at a distance of up to 200 meters and at a rate of 4-12 megabytes/s. [46].

Wi-Fi technologies are designed to provide access to wireless broadband networks for high speed data transfer. The networks can be expanded without interlayers and wire through $\mathrm{Wi}-\mathrm{Fi}$, with access to network and mobile devices. Within the Wi-Fi zone, several users can access the Internet on a computer, laptop, tablet, phone, etc. may be included in [47].

ZigBee (6LoWPAN) technologies. ZigBee is designed to create wireless personal networks (WPANs) using small-size radio transmitters with little power. ZigBee technology is oriented at the applications capable to operate separately and securely for a long time during high-speed data transmission [48-50].

ZigBee is used in bio-transmitters for medical diagnostic devices, medical equipment, and for the monitoring of the condition of athletes, including the personnel operating at high risk sites. In this case the maximum transmission speed accounts for $250 \mathrm{~kb} / \mathrm{s}$. ZigBee consumes low power operating in sleep mode. Devices can be enabled by pressing the button, working with the timer, and so on. "Sleeping" devices switch back to "sleep" mode as soon as the data is transmitted and they get the confirmation on the receipt of the package by the main line. The disadvantage of ZigBee technology is often due to the fact that it is not used on smartphones, although BLE is used. Therefore, the use of ZigBee technologies in fixed locations is recommended.

$\boldsymbol{A N T}+$ technology is a wireless communication standard designed to transmit information between ANT+ supported devices [7]. This standard uses the frequency used by Bluetooth, supports up to 30 meters distance, and is implemented through special chips allowing the data transmission between devices. The standard is intended for house use and medical application. This standard is used by Philips, Samsung, Sony, HbbTV, France Televisions. Its main advantages include low energy consumption, thus the ANT+ connection uses $70 \%$ less energy than Bluetooth.

3G/4G LTE (Long Term Evolution) and 5G technologies. 3G is a third-generation mobile communication technology, providing a set of services combining both high-speed mobile access technologies with data transmission channels, as well as Internet-based services. 3G networks work within the range of decimeters and centimeters, and transmit data at speeds of up to $3.6 \mathrm{mbit} / \mathrm{s}$.

$\boldsymbol{4 G} \boldsymbol{L T E}$ is a fourth-generation mobile communication technology, designed for high-speed wireless data transmissions in data-driven mobile devices and other equipment. The objective of 4G LTE is to increase speed and transmission capabilities using modulation methods and digital signal processing, as well as the reconstruction and simplification of IP-based network architecture, which will significantly reduce delays in data transmission in regard to $3 \mathrm{G}-$ architecture networks. 
$5 G$ is the next generation mobile communication technologies, which involves the creation of a network that practically enables connecting almost everything. The transition to global standard 5G NR (New Radio) will ensure a new mobile broadband connection for smartphones (tablets) in 2019. These devices sense the information from transmitters in the human body and send them to the network for general use [43, 51].

NFC technologies (Near Field Communication, NFC). In recent years, near field high-speed wireless technology has also improved significantly and allows data exchange between devices at a distance of about 10 centimeters. NFC technologies were primarily intended for the use on digital mobile devices. This technology is a simple expansion of contactless standards that connect interface smart cards considering it a single device. The NFC device supports the communication with a smart card and other NFC devices, which can work with existing contactless card infrastructures [8].

NB-IoT Technologies (Narrow Band IoT) is a new generation of cellular connection standards for telemetry devices for low-scale data exchange (2016), designed to connect a wide spectrum of autonomous devices, including medical devices to digital network connections [52]. Since NB-IoT is protected and supports a large number of devices and transmission at a great distance, it is very convenient for healthcare applications.

Transmitters. The development of transmitters is one of the main incentives for the expansion of the ICT application. Transmitters measure the physical data and convert them into raw information. This information is then stored digitally, and useful for analysis and processing.

Miniaturization of sensors has allowed them to integrate into "smart" devices, with the latter being able to record data, analyze the data, and allowing them to be transmitted over the Internet. The size of modern transmitters can range from one millimeter to tens of centimeters. At present, the work is underway to further reduce the dimensions of transmitters to ensure high comfort within the human body. Transmitters are fastened to the body in a variety of ways, and combined with the basic headset (often with a smartphone) via wireless technology, such as via Bluetooth, $A N T+$, ZigBee, etc.

Transmitters have to work perfectly and autonomously for the full realization of IoT capabilities, i.e., transmitters must be systematically fed. Solution of the problem should be sought from the environment: the methods of generating electricity from vibration, light and air flow [53]. Many achievements have already been done in this area. Scientists have announced the utilization of commercial nanogenerators - the chips that transform the movement of the human body (even one finger) into electrical energy, which avoids the use of battery and electrical sockets [54].

IoT platforms. An IoT device is interconnected via the Internet protocols for data transmission between each other. IoT-platforms provide bridge services between sensors and data transmission networks. The most popular companies in the IoT platform market may include:

- Amazon Web Services;

- Microsoft Azure;

- ThingWorx IoT Platform;

- IBM's Watson;

- Cisco IoT Cloud Connect;

- Salesforce IoT Cloud;

- Oracle Integrated Cloud;

- GE Predix.

\section{IoT and other technologies}

The development and use of IoT potential will be possible in its interaction with other technologies. Cloud technologies, Big Data tools and techniques, artificial intelligence technologies should be mentioned here first.

Cloud technologies. The IoT system generates a large amount of data to be stored, processed and shared. Clouds in the universal architecture of IoTs have three main functions: 
- data collection and storage (transmitters' indicators), and their accessibility. The devices with transmitters collect giant volumes of data; the latter ones are stored in the clouds for further processing and analysis;

- data analysis. Cloud services provide data review, cross-connections detection, and important data extraction, including transformation of transmitters' indicators for remote data exchange and decision-making. Real-time analytics (analytic processing), including the analytics implemented after packet mode data collection in wide intervals. In this case, machine learning and data acquisition algorithm and technology play an important role;

- providing execution commands: IoT systems refer to data in different directions, along with transmitting the transmitters' indicators, and ensuring that the commands are securely activated from the cloud.

In addition, cloud solutions perform administrative functions such as managing the records of device and users' logs, performing the protocols of use, monitoring of server status and reporting [55].

Big Data is one of the most important technologies that complements IoT and provides tools and techniques for large, diverse, different sized and unstructured data processing. The use of IoT also increases the number of large data in e-health [56]. Anyone in need of regular medical supervision and wearing a transmitter is the generator of infinite large numbers of digital anamnesis. Thus, one of the most important issues for the development of new medical technologies is the solution of large data generated by IoT transmitters. Modern medical technology allows scanning a body for only a second, whereas the whole human body is scanned for 60 seconds. This means that after appropriate examination, $10 \mathrm{gb}$ of data will be sent to disease archive in the form of unprocessed images and electronic reports. Moreover, the volume of data on the electronic health record (EHR) of an adult patient will account for more than 2 TB.

The development of specific analytical tools aimed at working with medical data is very important for the solution of big data problem in medical IoTs. Today Hitachi Clinical Repository's solutions are very successful in the market [57], these solutions allow processing the results of the examination and the raw medical data from various sources, and obtaining the necessary information.

Artificial intelligence technologies. Several researchers and developers offer creating "sensitive Internet objects" by transferring artificial intelligence to the "objects" and communications networks. They state that IoT system should have the features of "selfconfiguration, self-optimization, self-protection and self-healing" in the future [58, 59]. The "smart" things are estimated to be "smarter" due to context dependence, large memory, and extensive processing capabilities, as well as ability to think [60].

The Internet of Things generates a huge amount of data; however, the real problem is the timely and precise processing and analysis of this data. The analytical capabilities of artificial intelligence applications on servers that handle data obtained or served to the IoT networks can provide fast and adaptive data collection. The combination of IoT and artificial intelligence technologies will enable the development of "smart" and "connected" machines to interact with each other and to make decision with the without or minimized participation of a human in general.

\section{Conclusion}

IoT technologies allow collecting and processing relevant data, analyzing them, and detecting potential problems along with the monitoring of the health and location of the personnel on SP. Thus, the data received from smart devices during a certain period of time allows building the behavioral patterns of the personnel on SP and providing the staff with clinical information based on the discrepancies recorded by the health monitoring results. One of the trends promoting the realization of these capabilities is the gradual reduction of the size of the connecting devices, which enhances the capability of portable devices to be implanted to the human body. In this case, microscopic transmitters installed in the human body (inside) and body-worn devices allow the collection of objective information and the control of the treatment process (at any point of the 
world). This increases the probability of the provision of medical care before undesirable and unpleasant situations occur.

Today, many countries recognize that IoT is capable to radically change human resources management in the world as a whole, as well as in certain areas, particularly those at risky sites. Unquestionably, many countries are developing the strategies and guidelines for installing IoT technologies at special risk sites (coal mines, mines, oil platforms, etc.), and conducting research on different segments of IoTs. It should be noted that the use of IoT to monitor the status and to track the location of the personnel on SP is still at the onset of development. At this stage of development, along with the development and expansion of appropriate IoT solutions, the profound understanding of the essence of these technologies should be achieved and consumers' perspectives on understanding their benefits should be changed.

\section{Acknowledgements}

This work was financially supported by the Science Fund of the State Oil Company of Azerbaijan Republic (SOCAR EF 2017).

\section{References}

1. SOCAR reports. www.socar.az/socar/az/economics-and-statistics/economics-and-statistics/socar-reports

2. GOST R 54594-2011. Platform of marine. Rules of habitability. General requiremends. http://docs.cntd.ru/document/gost-r-54594-2011

3. The Internet of Things (IoT) - essential IoT business guide. www.i-scoop.eu/internet-ofthings-guide/.

4. Gringard S. Internet of things: The future is already here. Ed. Alpina Pablisher, 2016, 188 p.

5. Khan R., Khan, S.U., Zaheer R., Khan S. Future Internet: The Internet of Things Architecture, Possible Applications and Key Challenges, 2012. https://pure.qub.ac.uk/portal /files/ 81384964/ PID2566391.pdf.

6. Roslyakov A.V., Vanyashin S.V., Grebeshkov A.Yu. Internet of things, Samara, 2015, 136 p.

7. Rose K., Eldridge S., Chapin L. Internet of Things: brief overview, 2015, 78 p.

8. Zhirkov A. Internet of Things and Cloud Technologies Eurotech. www.cta.ru/cms/f/460221.pdf.

9. Report-Internet of Things-2015. www.internetsociety.org/wp-content/uploads/2015/10/ report-InternetOfThings-20151221-ru.pdf

10. James M., Chui M., Bisson P., Woetzel J., Dobbs R., Bughin J., Aharon D. The Internet of Things: Mapping the Value Beyond the Hype. McKinsey Global Institute, June 2015, 144 p. www.mckinsey.com/mgi

11. Plaza I., Martín L., Martin S., Medrano C. Mobile applications in an aging society: Status and trends // The Journal of Systems and Software, 2011, vol.84, no.11, pp.1977-1988.

12. Review of the Internet of Things, ITU Recommendation, Y.2060, 2012, No 6, 22 p.

13. Ko J., Lu C., Srivastava M. B., Stankovic J. A., Terzis A., Welsh M. Wireless sensor networks for healthcare // Proceedings of the IEEE, 2010, vol.98, no.11, pp.1947-1960.

14. Alemdar H., Ersoy C. Wireless sensor networks for healthcare: A survey // Computer Networks: The International Journal of Computer and Telecommunications Networking, 2010, vol.54, no.15, pp.2688-2710.

15. Appelboom G., Camacho E., Abraham M.E., Bruce S.S., Dumont E.L., Zacharia B.E., D’Amico R., Slomian J., Reginster J.Y., Bruyère O., Connolly E.S. Smart wearable body sensors for patient self-assessment and monitoring // Archives of Public Health, 2014. https://doi.org/10.1186/2049-3258-72-28.

16. Aliverti A. Wearable technology: role in respiratory health and disease // Breathe, 2017, vol.13, no.2, pp.27-36. http://doi.org/10.1183/20734735.008417. 
17. Glossary on the Information Society / Under Ed. Yu.E. Khokhlova, Moscow: Institute for the development of the Information Society, 2009, $162 \mathrm{p}$.

18. Watkins P.J. ABC of Diabetes, 2nd edition, Moscow: Binom, 2006, 134 p.

19. Gia T.N., Ali M., Dhaou I.B., Rahmani A.M., et.al. IoT-based continuous glucose monitoring system: A feasibility study // Procedia Computer Science, 2017, vol.109, pp.327-334.

20. Istepanian R.S.H., Hu S., Philip N.Y., Sungoor A. The potential of Internet of m-health Things ' $\mathrm{m}-\mathrm{IoT}$ ' for non-invasive glucose level sensing / Proc. IEEE Annu. Int. Conf. Eng. Med. Biol. Soc. (EMBC), Aug./Sep. 2011, pp.5264-5266.

21. Guan Z. J. Somatic data blood glucose collection transmission device for Internet of Things, Chinese Patent 202838653U, 2013.

22. Monitoring the state of the cardiovascular system. What is ECG, how to decode it independently. http://okardio.com/diagnostika/ekg-201.html

23. Castillejo P., Martinez J.-F., Rodriguez-Molina J., Cuerva A. Integration of wearable devices in a wireless sensor network for an e-health application // IEEE Wireless Commun., 2013, vol.20, no.4, pp.38-49.

24. Agu E. et al. The smartphone as a medical device: Assessing enablers, benefits and challenges / Proc. IEEE Int. Workshop Internet-Things Netw. Control (IoT-NC), Jun. 2013, pp.48-52.

25. Liu M.-L., Tao L., Yan Z. Internet of Things-based electrocardiogram monitoring system, Chinese Patent 102764118 A, Nov.7, 2012.

26. Xiaogang Y., Hongjiang L., Jiaqing W., Wentao T. Realization of comprehensive detection algorithm of electrocardiogram signal at application layer electrocardiogram monitoring Internet of Thing, Chinese Patent 101947112 A, Jan.19, 2011.

27. Ortiz K.J.P., Davalos J.P.O., Eusebio E.S., Tucay D.M. IoT: Electrocardiogram (ECG) Monitoring System // Indonesian Journal of Electrical Engineering and Computer Science, 2018, vol.10, no.2, pp.480-489.

28. Fabregat-Andres O., Munoz-Macho A., et.al. Evaluation of a New Shirt-Based Electrocardiogram Device for Cardiac Screening in Soccer Players: Comparative Study With Treadmill Ergospirometry // Cardiology Research, Elmer Press Inc., 2014, vol.5, no.3-4, pp.101-107.

29. Tarouco L.M.R. et al. Internet of Things in healthcare: Interoperatibility and security issues / Proc. IEEE Int. Conf. Commun. (ICC), Jun. 2012, pp.6121-6125.

30. Guan Z.J. Internet-of-Things human body data blood pressure collecting and transmitting device, Chinese Patent 202821362U, Mar. 27, 2013.

31. Xin T.J., Min B., Jie J. Carry-on blood pressure/pulse rate/blood oxygen monitoring location intelligent terminal based on Internet of Things, Chinese Patent 202875315U, Apr.17, 2013.

32. Ruiz M.N., García J.M., Fernández B.M. Body temperature and its importance as a vital constant // Revista Enfermeria, 2009, vol.32, no.9, pp.44-52.

33. In Z.L. Patient body temperature monitoring system and device based on Internet of Things, Chinese Patent 103577688A, Feb.12, 2014.

34. Mosa A.S.M., Yoo I., Sheets L. A systematic review of healthcare applications for smartphones // BMC Med. Informat. Decision Making, 2012, vol.12, p.67.

35. Islam R.S.M., Kwak D., Kabir M.H., Hossain M., Kwak K.S. The Internet of Things for Health Care:A Comprehensive Survey // IEEE Access, 2015. DOI 1 0.1109/ACCESS.2015.2437951

36. White P.J.F., Podaima B.W., Friesen M.R. Algorithms for smartphone and tablet image analysis for healthcare applications // IEEE Access, 2014, vol.2, pp.831-840.

37. Zaramenskikh E.P., Isaev E.A., Korovkina N.L. Intellectualization of information processing in the system of electronic medical monitoring // Mathematical Biology and Bioinformatics, 2016, T. 11, No 2, pp 288-298.

38. GPS tracking. https://whatis.techtarget.com/definition/GPS-tracking 15.

39. Ngai E.W.T., Moon K.K., Riggins F.J., Yi C.Y. RFID research: An academic literature review 
(1995-2005) and future research directions // International Journal of Production Economics, 2008, vol.112, no.2, pp.510-520.

40. Mohd I.B., Shariq A., Asif A., Suhail A. E-Health with Internet of Things // International Journal of Computer Science and Mobile Computing, IJCSMC, 2017, vol.6, no.6, pp.357-362.

41. Smirnov A. RFID can save more than 7 thousand lives. www.cnews.ru/reviews/free/ publichealth2010/article/article8.shtml

42. Sana U., Henry H., Bart B., Benoit L., et al. A Comprehensive Survey of Wireless Body Area Networks // Journal of Medical Systems, 2012, vol.36, no.3, pp.1065-1094.

43. Fam V.D., Kirichek R.V., Glushakov R.I., Pirmagomedov R.Ya. Internet of things technologies for health applications // Information Technologies and Telecommunications, 2017, vol. 5, No4, pp.71-77.

44. He W., Xu L. Integration of distributed enterprise applications: A survey // IEEE Transaction on Industrial Informatics, 2014, vol.10, no.1, pp.35-42.

45. Uckelmann D., Harrison M., Michahelles F. An architectural approach towards the future internet of things // Architecting the Internet of Things USA, NY: Springer, 2011, pp.1-24.

46. www.russianelectronics.ru/leader-r/review/2187/doc/58627/Инт_вещей2017ВКР3372

47. www.russianelectronics.ru/leader-r/review/2187/doc/58627/]

48. Fahier N., Fang W.-C. An Advanced Plug-and-Play Network Architecture for Wireless Body Area Network Using HBC, ZigBee and NFC // IEEE International Conference on Consumer Electronics (ICCE), 2014. pp.165-166.

49. Höller J., Tsiatsis V., Mulligan C., Karnouskos S., Avesand S., Boyle D. From Machine-toMachine to the Internet of Things: Introduction to a New Age of Intelligence. Amsterdam, The Netherlands: Elsevier, 2014, 352 p.

50. Li Q., Wang Z., Li W., Li J., Wang C., Du R. Applications integration in a hybrid cloud computing environment: Modelling and platform // Journal Enterprise Information Systems, 2013, vol.7, no.39. https://doi.org/10.1080/17517575.2012.677479.

51. Searching for the first "killer" 5G use case? Reach inside your pocket! www.qualcomm.com/news/onq/2017/05/04/first-killer-5g-use-case-inside-your-pocket

52. 3GPP Low Power Wide Area Technologies. GSMA white paper, 2016, 49 p. www.gsma.com/iot/wp-content/uploads/2016/10/3GPP-Low-Power-Wide-Area-

Technologies-GSMA-White-Paper.pdf

53. Yee W.S., Yung C.L. Smart Dust Sensor Network with Piezoelectric Energy Harvesting, ICITA, 2009. www.icita.org/papers/34-sg-Liang-217.pdf

54. Dave E. Internet of things. How will our whole life change at the next stage of development of the World Wide Web. 2011, 14 p. www.cisco.com/c/dam/global/ru_ru/assets/ executives/pdf/internet_of_things_iot_ibsg_0411final.pdf

55. Taivasaari A., Mikkonen T. Features of software development in the era of the Internet of things. Open systems. DBMS, 2017, No. 02. www.osp.ru/os/2017/02/13052220/

56. Mammadova M.G. Big Data in electronic health: opportunities, challenges and prospects // Information Technology Problems, 2016, No2, pp.9-29.

57. Hitachi Clinical Repository. www.hitachimed.com/idc/groups/hitachimedical/documents/ supportingdocumentpdf/ poc_021435.pdf

58. Arsénio A., Serra H., Francisco R., Nabais F., Andrade J., Serrano E. Internet of Intelligent Things: Bringing artificial intelligence into things and communication networks // Stud. Comput. Intell., 2014. vol.495, pp.1-37. doi.org/10.1007/978-3-642-35016-0_1

59. Kortuem G., Kawsar F., Fitton D., Sundramoorthy V. Smart objects as building blocks for the internet of things // IEEE Internet Comput., 2010, vol.14, no.1, pp.30-37.

60. Ding Y., Jin Y., Ren L., Hao K. An intelligent self-organization scheme for the internet of things // IEEE Comput. Intell. Mag., 2013, vol.8, no.3, pp.41-53. 